\title{
LA-UR-96- 3172
}

Title:

Ion Flooding to Precharge Insulating Materials

Author(s):

Duncan MacArthur, NIS-6

Peter Steadman, NIS-6

John Bounds, NIS-6

Charles Whitley, NIS-6

Mohini Rawool-Sullivan, NIS-6

Submitted to:

DOE Office of Scientific and Technical Information (OSTI)

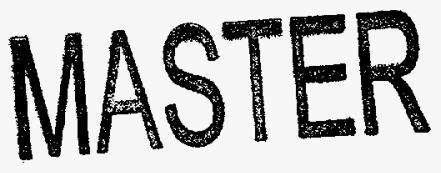

Los Alamos

NATIONAL LABORATORY

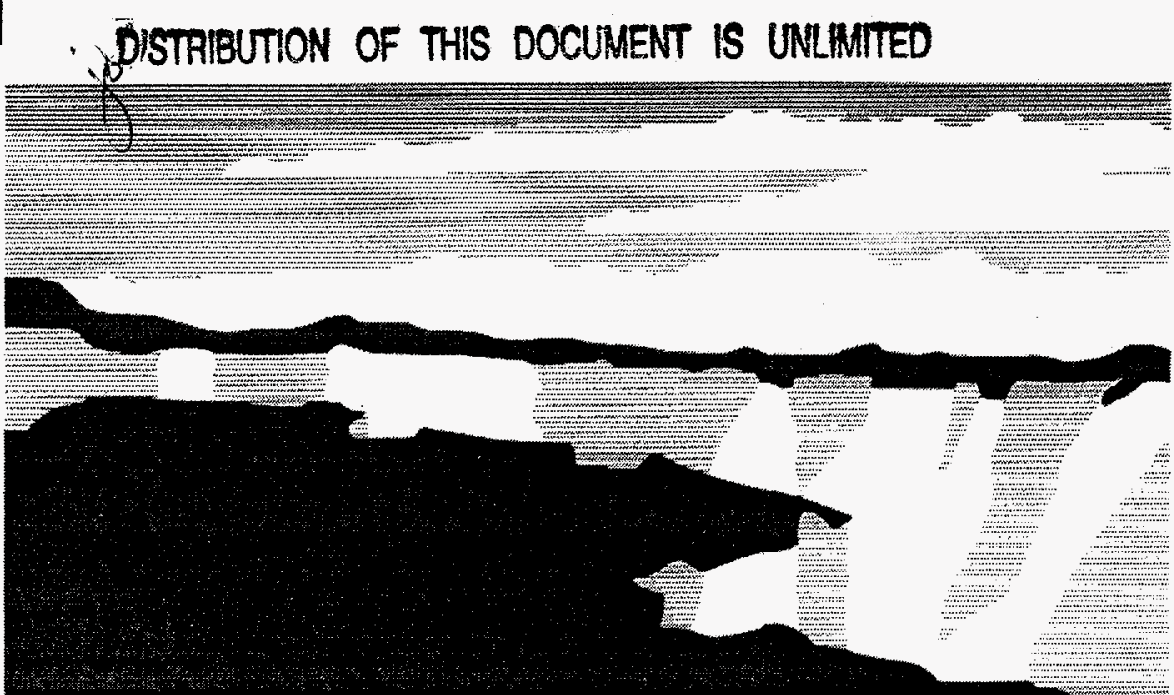

Los Alamos National Laboratory, an aftirmative action/equal opportunity employer, is operaled by the University of California for the U.S. Department of Energy under contract W-7405-ENG-36. By acceptance of this article, the publisher recognizes that the U.S. Government retains a nonexclusive, royaltyfree license to publish or reproduce the published form of this contribution, or to allow others to do so, for U.S. Government purposes. The Los Alamos National Laboratory requests that the publisher identify this article as work performed under the auspices of the U.S. Department of Energy. 


\section{DISCLAIMER}

Portions of this document may be illegible in electronic image products. Images are produced from the best available original document. 


\section{DISCLAIMER}

This report was prepared as an account of work sponsored by an agency of the United States Government. Neither the United States Government nor any agency thereof, nor any of their employees, makes any warranty, express or implied, or assumes any legal liability or responsibility for the accuracy, completeness, or usefulness of any information, apparatus, product, or process disclosed, or represents that its use would not infringe privately owned rights. Reference herein to any specific commercial product, process, or service by trade name, trademark, manufacturer, or otherwise does not necessarily constitute or imply its endorsement, recommendation, or favoring by the United States Government or any agency thereof. The views and opinions of authors expressed herein do not necessarily state or reflect those of the United States Government or any agency thereof. 


\title{
Ion Flooding to Precharge Insulating Materials
}

\author{
Duncan MacArthur*, Peter Steadman, John Bounds, Charles Whitley, \\ and Mohini Rawool-Sullivan
}

\begin{abstract}
This is the final report of a one-year, Laboratory-Directed Research and Development (LDRD) project at the Los Alamos National Laboratory (LANL). The collection of ions created by alpha particles in air is a powerful technique for detecting the presence of alpha-emitting radioactive contamination. These ions have a relatively long lifetime (greater than 5 seconds) in air so the ions can travel much longer distances than the alpha particles themselves. However, such ion detection is difficult when the object being surveyed is an insulating material. An insulating object does not release ions generated until the surface of the object has completely discharged. Thus, there are significant responsetime problems with contamination on insulating objects. This project sought to investigate the technique of "flooding" the suspect object with ions prior to the actual contamination detection measurement. Our preliminary conclusion is that such flooding will enhance detection speed on insulating objects.
\end{abstract}

\section{Background and Research Objectives}

The long-range alpha detection (LRAD) technology $[1,2]$ has been developed for use in the areas of detection and assay of radioactive contamination (particularly alpha-emitting contamination). Although we have detailed many potential applications of the LRAD technique, $[3,4]$ questions remain concerning the effects of charged insulators on the ion populations in gases. All detectors based on the LRAD technique collect the ions that are produced in the air by the interaction of alpha particles (produced by the decay of alphaemitting isotopes) with ambient air molecules [1-4]. These ions are transported to a collection electrode by an air current [5] or an electrostatic field [6]. The signal produced on the electrode consists of the very small current that is produced when these ions flow to ground. The detector's ability to collect ions, and therefore the detector's efficiency for alpha particle

\footnotetext{
* Principal investigator, e-mail: dmacarthur@lanl.gov
} 
detection, is hampered by stray electric fields produced by charges that have collected on the surfaces of insulators.

In some applications, it is possible to minimize insulating materials in areas where ions are generated. However, for many it is not possible. One in particular-one for which LRAD technology is especially well suited-is severely affected by the problems associated with insulating materials. Tools, electronic modules, laboratory apparatus, etc., are difficult to monitor by conventional methods, but lend themselves to monitoring by LRAD-based detectors. LRAD-based systems called object monitors, intended specifically for this monitoring application, have been successfully developed at Los Alamos and commercial models have been built by Eberline Instrument Corp. A solution to the problems posed by insulating materials is necessary if object monitoring is not to be restricted to metallic objects.

There are two types of LRAD detectors that are distinguished by the method used to gather the air ions. In airflow-type LRAD monitors [5], an airflow is established in which the detection electrode is situated downwind of the object being interrogated, as shown in Figure 1. In electrostatic-type LRAD monitors [6], an electric field transports the ionized air molecules to the detection electrode. In both types of monitor, the electric field associated with the charge that can build up on insulating surfaces competes with the ion-transport process. The detector efficiency then depends inversely on the amount of charge carried by the insulator. Because the effect is somewhat more pronounced in airflow-type detectors, all of the tests described in this report were performed using a simple airflow LRAD monitor.

Though the signal suppression will eventually disappear as the residual electrostatic charge is neutralized, the sensitivity suppression can last for several hours and be quite dramatic as shown in Figure 2. The proposed solution investigated in this project was to neutralizing any charge on the insulator by flooding the surrounding air with ions (of both polarities) prior to taking a measurement.

\section{Importance to LANL's Science and Technology Base and National R\&D Needs}

This project supports Los Alamos core competencies in analysis and assessment as well as earth and environmental systems. Although the ion flooding technique investigated here is not the entire LRAD technology, the ability to apply LRAD techniques to a new class of problems can provide a better understanding of the LRAD detection phenomenon and increases the opportunities for further use of these detection systems. 


\section{Scientific Approach and Results to Date}

We made seven 3-inch by 6.25 -inch swatches of test materials: two from protective gloves ("Pylox" and "Nitrile"), one made of plastic vinyl electrical tape, one from regular white typing paper, one from an "anti-c" bootie, and two of poly/cotton woven material, the first from a pair of yellow "anti-c" coveralls and the other from a white lab coat. We rubbed these vigorously to generate a static charge and then introduced them into the detection volume of an airflow-type object monitor along with an 11,500-dpm, plutonium-239 check source. The data depicted in Figure 2 was generated during one such test. We chose the poly/cotton swatch from the yellow "anti-c" coveralls for the subsequent tests because it accepted a charge more easily and was therefore more conducive to reproducibility.

As a measure of the effect of the static charge on the ability to detect ions, we used the time it took for the signal to rise from $10 \%$ to $90 \%$ of its final value. Although we expected this rise time to depend to a large extent on the humidity, our data is inconclusive about the relationship between humidity and rise time, and indicates that there are other, presently unknown, factors that control the rise time. To test the effect of humidity, we repeated a test with the yellow swatch nine times over a period of two weeks during which the humidity varied. The test, as before, involved rubbing the swatch to apply a charge, then monitoring the plutonium source while it sat on the swatch. The data shows only weak, if any, correlation between rise time and humidity, as seen in Figure 3.

Our first attempts at ion flooding utilized two different commercial ion generators. Neither succeeded in neutralizing the charge because they both provided an inadequate balance between populations of positive and negative ions. Ion population imbalances bring the charge on the insulators to some set level that depends on the degree of the imbalance. We found that these generators actually applied charge to previously discharged insulators rather than discharging them. This type of ion generator produces each species of ion with different electrodes. In order to provide balanced populations, the electric field configuration around each electrode must be the same. For this reason, it is difficult for inexpensive generators to provide truly balanced ion populations when located inside of a metal box such as the airflowtype detector. In order for the populations to be balanced, the two electrodes need to be situated symmetrically with respect to all sides of the box. The difference in relative size of the two populations was found to depend quite sensitively on the placement of the generator in the detector chamber. Because of this sensitivity and the time required for measurements, the electrostatic ion generator approach was abandoned in favor of a radioactive ion source. Radioactive sources, which generate ions by through ionizing radiation, intrinsically provide a perfectly balanced ion population. Electronic sources would be preferable, however, for a final 
solution in commercial LRAD detectors. Manufacturers would prefer not to load a commercially available detection device with large amounts of radioactive material. The use of a radioactive ion source for this study was intended only as a proof of principle.

Figures 4 and 5 are sketches that show how the ion flooding technique was implemented in the air-flow type object monitor using a radioactive ion source. Four Static Master $®$ refill units were used as the ion source. Static Masters ${ }^{\circledR}$ are anti-static and anti-dust brushes that are intended mainly for use in photography. Each one contains approximately $500 \mu \mathrm{Ci}$ of polonium-210; the ion cloud generated by the alpha radiation from the polonium is used to eliminate the static charge on film or other surfaces so that dust motes, which adhere to charged surfaces, can be removed by the brush. Each refill unit has two strips of polonium metal contained in a small aluminum cage. Four of these refill units were fixed to the ceiling of the detector and encased in pockets made of conductive foam (bottom sketch in Figure 4). Pieces of conductive foam were also fixed to the blades of a ceiling-fan-shaped covering device (top sketch in Figure 4) so that the ion sources could be sealed off while measurements were taken. The four covers were suspended in the middle by a metal rod so that the Static Masters® could be sealed off and exposed by rotating the rod. This system allows the detector chamber to be flooded with ions without air flow disturbances caused by opening the detector door. There was also a 24-Volt direct current fan suspended in the approximate center of the detector chamber. When the sources were exposed, the detector fans - the fans which carry the air over the object and onto the detection electrodes (c.f. Figure 1)-were turned off and the fan suspended in the middle of the chamber was turned on. This fan carried the ionization cloud generated by the exposed polonium sources down onto the swatch.

Quantifying the results was complicated by the variability of the rise times. Attempts were be made to isolate the detector from the main factors that control the rise time, so that effects due to the time duration the polonium sources are exposed, the speed of the 24-Volt direct current fan, and the amount of radioactive material could be explored. The influence of these variables has not been investigated.

Tests such as the one illustrated in Figure 6 have made it clear that the ion flooding solution is effective. In a test similar to that depicted in Figure 2, a static charge was applied to the same poly/cotton swatch used in previous experiments. The polonium sources were exposed for approximately one minute. After the exposure, the signal rose from approximately $50 \%$ of its final value to approximately $95 \%$ of its final value. By extrapolation of the curve preceding the exposure, we infer that the signal would not have achieved this rise for at least ten minutes. In this test, a one-minute exposure decreased the rise time by around $30 \%$; however, it is not clear whether the quantity that is affected by a given exposure is a percentage decrease of the rise time or simply the amount of charge on the insulator. 


\section{References}

1. MacArthur, D. W., K. S. Allander, J. A. Bounds, and K. B. Butterfield, "Small LongRange Alpha Detector (LRAD) with Computer Readout," Los Alamos National Laboratory publication LA-12199-MS (1991).

2. MacArthur, D. W., K. S. Allander, J. A. Bounds, M. M. Catlett and J. L. McAtee, "Long-Range Alpha Detector for Contamination Monitoring", IEEE Transactions on Nuclear Science, 39, 952 (1992).

3. Rutherford, "Alpha Contamination Monitoring of Surfaces, Objects, and Enclosed Areas," IEEE Transactions on Nuclear Science, 40, 840 (1993).

4. Steadman, P. A., J. D. Johnson, J. P. Johnson, J. E. Koster, K. Olsen, K. S. Allander, J. A. Bounds, and D. W. MacArthur, "A Portable Sample Monitor for LowLevel Alpha Contamination," in Proceedings of WM'95, Tucson AZ, February 26 -March 2, 1995.

5. Caress, R. W., K. S. Allander, J. A. Bounds, M. M. Catlett, and D. W. MacArthur, "LRAD-Based Airflow Monitors," Los Alamos National Laboratory publication LA12742-MS (1994).

6. MacArthur, D. W., K. S. Allander, J. A. Bounds, M. M. Catlett, R. W. Caress, and D. A. Rutherford, "LRAD Surface Monitors," Los Alamos National Laboratory publication LA-121524-MS (1993). 


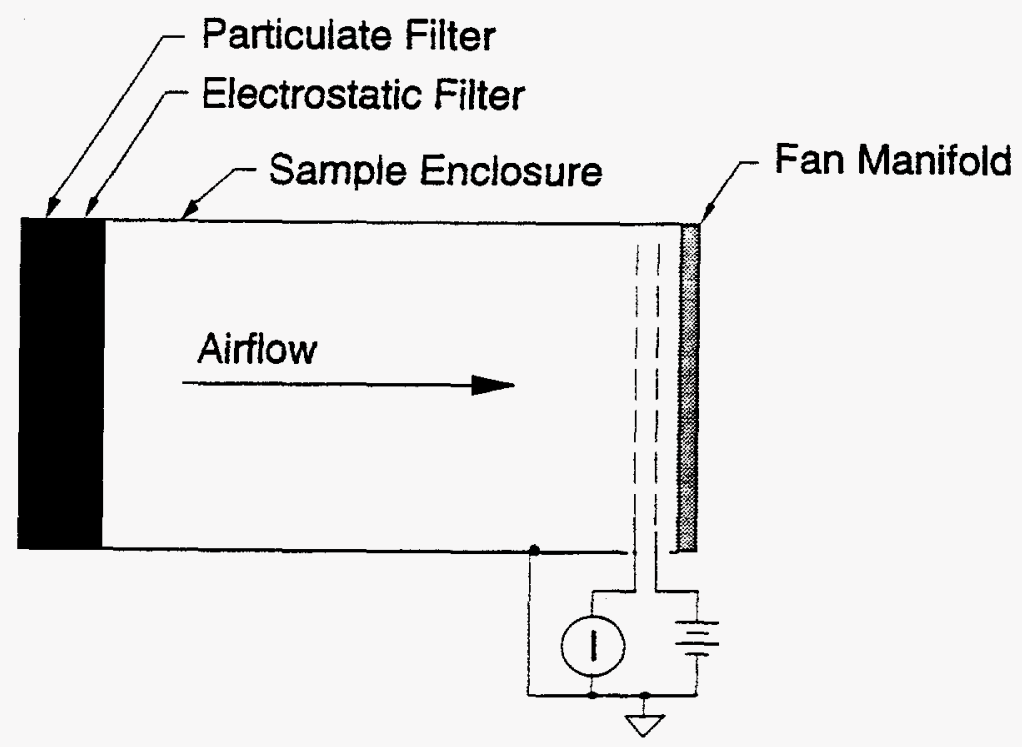

Figure 1. A generalized airflow-type LRAD design.

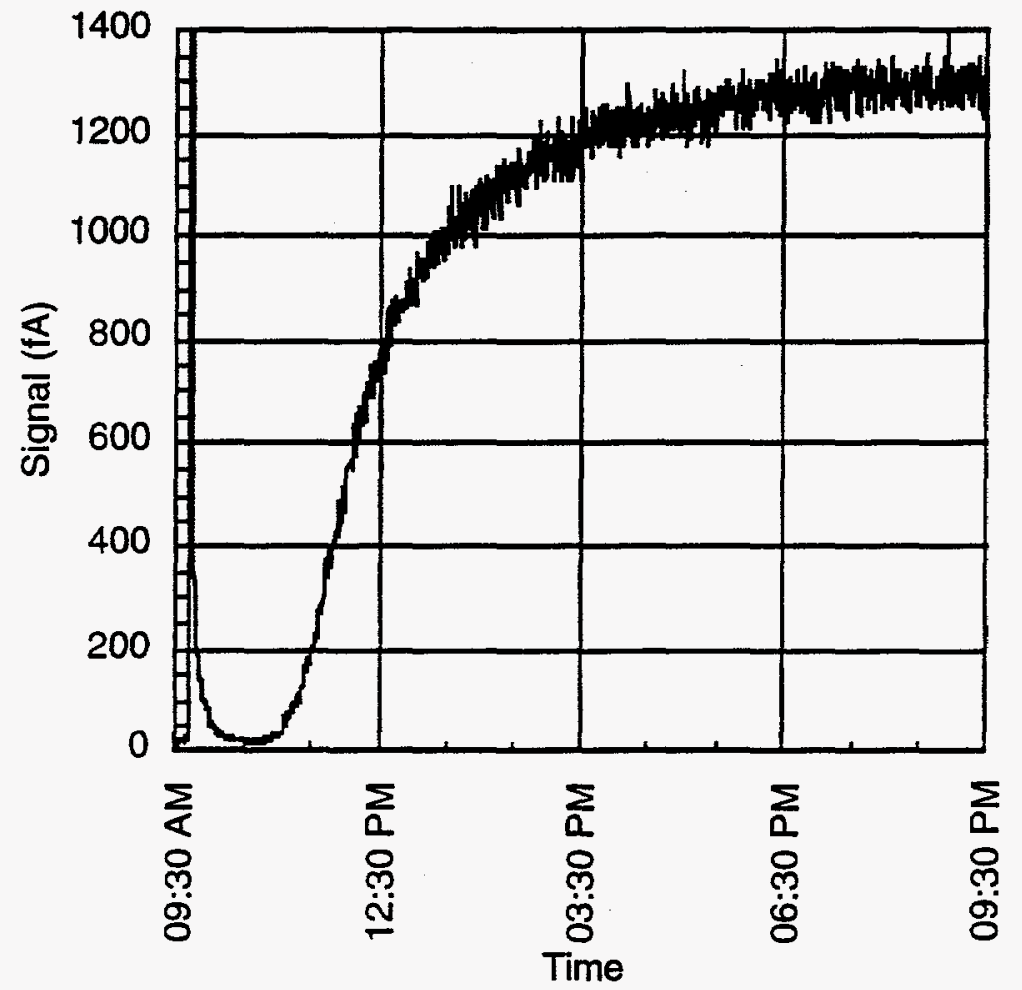

Figure 2. Response of an airflow-type object monitor to $11,500 \mathrm{dpm}$ of plutonium-239 in the presence of a charged swatch of poly/cotton material. 


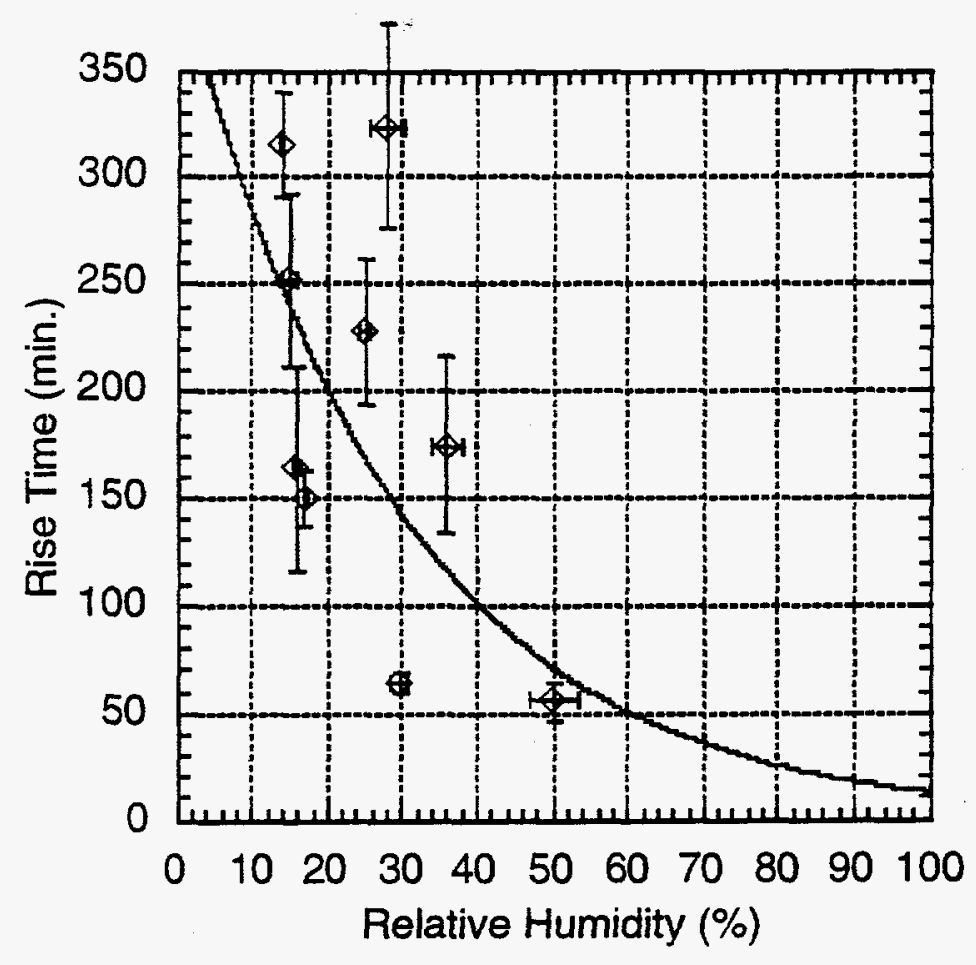

Figure 3. Rise time (time to go from $10 \%$ to $90 \%$ of final signal) plotted against humidity.

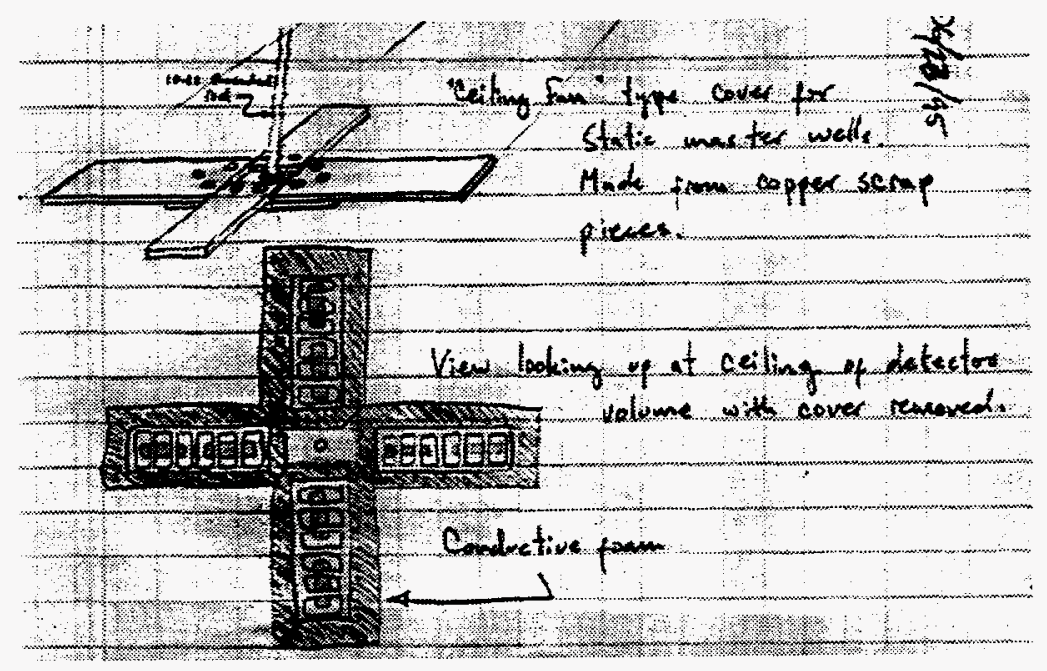

Figure 4. Schematics of the ion flooding method used in an airflow-type object monitor. 


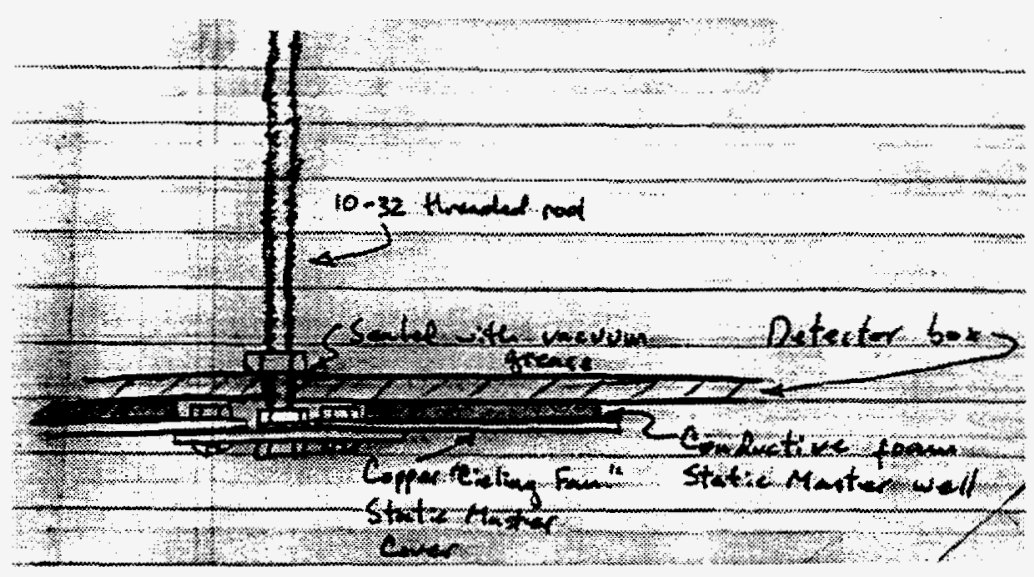

Figure 5. Schematics of the ion flooding method used in an airflow-type object monitor.

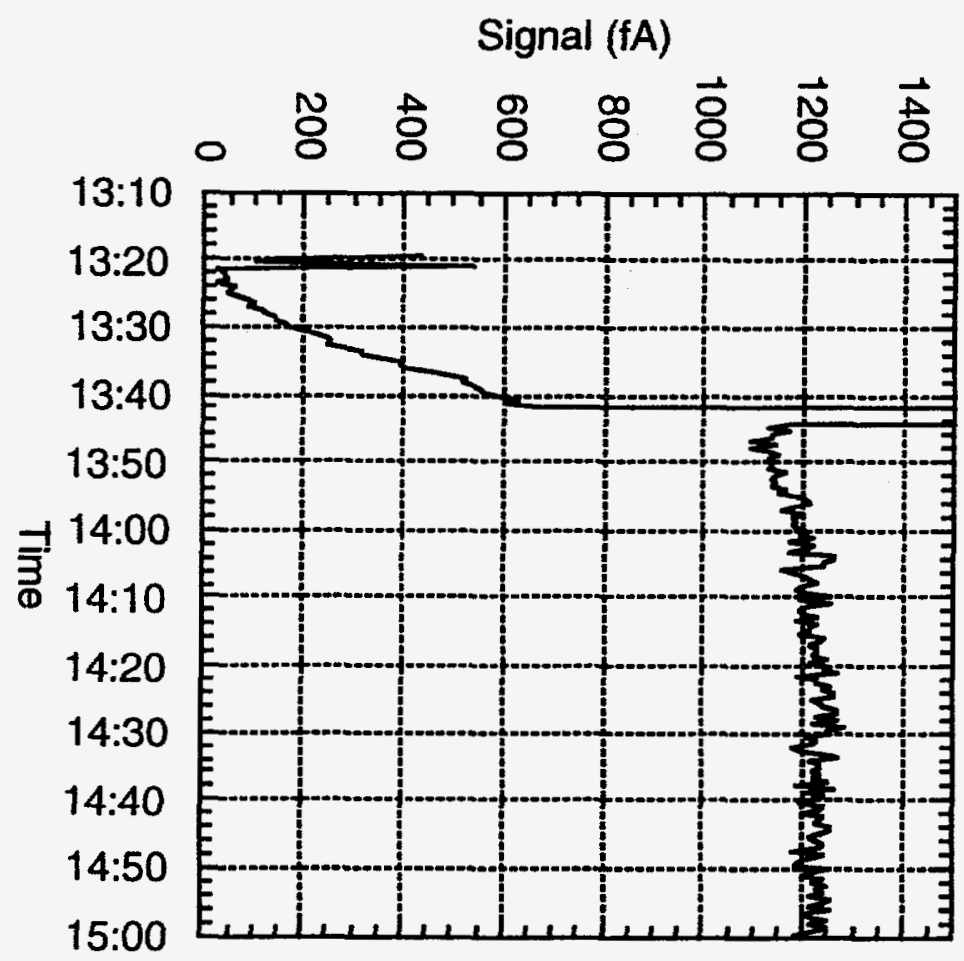

Figure 6. The effect of radioactive source ion flooding on the ability of a poly/cotton swatch to suppress the signal generated by an $11,500 \mathrm{dpm}$ plutonium-239 check source. The polonium-210 sources were exposed for approximately one minute starting at $13: 42$. 